\title{
Verificando a compreensão do BPMN com gestores de negócio
}

\author{
Vanessa Yadira Mendoza ${ }^{1}$ \\ Denis Silva da Silveira ${ }^{2}$
}

\begin{abstract}
Resumo: Engenheiros, artistas e artesões têm criado modelos há muito tempo para experimentar projetos antes de executá-los. Isso faz com que as ideias e soluções propostas nos modelos se tornem claras o suficiente para serem percebidas ou entendidas. De forma análoga, a utilização dos modelos de processos de negócio pode contribuir para a especificação dos requisitos de software, facilitando o entendimento e a comunicação do negócio do ponto de vista dos projetistas de software, bem como dos gestores. No entanto, há poucas pesquisas que examinam se os modelos de processos de negócio em BPMN são mais eficazes em sua compreensão do que outras representações, como por exemplo, as descrições textuais. Este artigo apresenta uma pesquisa realizada com pessoas da área de gestão, que conhecem e que não conhecem BPMN, para verificar se há diferenças significativas em termos da compreensão dos modelos BPMN em comparação com uma representação textual. Os resultados encontrados mostram que a compreensão dos modelos BPMN pode refletir uma perda de comunicação entre os gestores, aqueles que entendem do negócio, e os desenvolvedores, profissionais da área de computação que consolidam os processos em requisitos de software.
\end{abstract}

Palavras-chave: BPMN, Compreensão, Processos de negócios.

\begin{abstract}
Engineers, artists and craftsmen have long-established models to try out projects before running them. This makes the ideas and solutions proposed in the models clear enough to be perceived or understood. Similarly, the use of business process models can contribute to the specification of software requirements, facilitating the understanding and communication of the business from the point of view of software designers, as well as the managers. However, there is little research that examines whether BPMN business process models are more effective in their understanding than other representations, such as textual descriptions. This paper presents a research carried out with people from the management field, some who are familiar with and some who do not know BPMN, to verify if there are significant differences in terms of understanding the BPMN models in comparison to a textual representation. The results show that the understanding of the BPMN models can reflect in a loss of communication between the managers, the ones who understand the business, and the developers, professionals of the area of computer science who consolidate the processes in software requirements.
\end{abstract}

Keywords: BPMN, Understanding, Business Process.

\section{Introdução}

A modelagem de processos é uma prática que vem sendo adotada pelas organizações nos contextos mais diversos. Modelos de processos de negócio podem e são utilizados para diversos fins, como a revisão de melhores práticas, redefinição de estratégias de negócio e especificação de requisitos de sistemas de informação

\footnotetext{
${ }^{1}$ Pós-Graduação em Engenharia da Computação, Universidade de Pernambuco (UPE)

\{vymz@ecomp.poli.br\}

${ }^{2}$ Departamento de Ciências Administrativas - Universidade Federal de Pernambuco (UFPE)

\{dsilveira@ufpe.br
}

http://dx.doi.org/10.5335/rbca.v9i4.7076

Revista Brasileira de Computação Aplicada (ISSN 2176-6649), Passo Fundo, v. 9, n. 4, p. 60-75, dez. 201760 
[1]. Segundo Vanderfeesten et al. [2], existe uma forte analogia entre sistemas de informação e processos de negócios. Um sistema de informação é geralmente dividido em módulos ou funções, isto é: em atividades que recebem entradas (insumos) e proporcionam saídas (produtos) a partir de um processamento. Semelhante a essa estrutura de composição, um modelo de processo de negócio consiste em um conjunto estruturado de atividades ordenadas no tempo e no espaço, que produz um produto ou um serviço com início e fim, e com entradas e saídas bem definidas.

Todavia, independente da definição utilizada para um modelo de processos, a sua compreensão por todos os seus stakeholders é de suma importância para as organizações. Corroborando com essa afirmação, Reijers e Mendling [3] afirmam que um dos principais objetivos de um modelo de processo é facilitar a comunicação entre os stakeholders. No entanto, ainda segundo esses autores, pouco se sabe sobre os fatores que realmente influenciam a compreensão de um modelo de processo pelos agentes humanos.

Segundo Laue e Gadatsch [4], a compreensão é um critério que ajuda a mensurar se as informações contidas em um modelo podem ser entendidas (ou compreendidas) por um leitor. Os autores destacam, ainda, que a compreensão é um dos critérios para a avaliação da qualidade de um modelo. Por outro lado, quando se fala em modelagem de processos, a notação de modelagem de processos de negócio (do inglês: Business Process Model and Notation ou BPMN) [5] é um padrão. Neste ponto é interessante ressaltar que o BPMN é apresentado por alguns autores (Tian et al. [6]; Martínez-Salvador et al. [7]; Istoan [8]; Geambasu [9]; Fernández et al. [10]; Schrepfer et al. [11]; Wahl e Sindre [12]) como sendo uma notação intuitiva e de fácil compreensão para os seus usuários, facilitando significativamente a integração entre os profissionais envolvidos, tais como os profissionais da área de gestão, especialistas do negócio e os da área de tecnologia. Mas, será que isso é realmente um fato?

Até aonde foi revisado na literatura, não há nenhum indício (investigação) que apresente uma verificação para o nível de compreensibilidade dos modelos em BPMN com especialistas ou gestores do negócio. Por esse motivo, o presente artigo tem como objetivo verificar se esse grupo de usuários (profissionais da área de gestão) possuem a correta compreensão dos modelos de processos de negócio representados em BPMN. Nesse sentido, este artigo realizou uma comparação, entre um processo de negócio descrito em texto e modelado BPMN, para verificar a real compreensão dos usuários da área de negócio.

Além desta seção introdutória, este artigo foi organizado da seguinte maneira: a seção 2 apresenta o referencial teórico, retratando a compreensão de modelos, gestão de processos de negócio e BPMN, a seção 3 apresenta os procedimentos metodológicos utilizados para a realização da pesquisa, a seção 4 apresenta análise e a discussão da pesquisa em relação aos resultados encontrados; a seção 5 apresenta os trabalhos relacionados e, por fim, a seção 6 com as considerações finais.

\section{Referencial Teórico}

Esta seção apresenta os conceitos da literatura considerados importantes para operacionalização da fase empírica da pesquisa. Assim, a presente seção foi estruturada em três tópicos: compreensão de modelos, gestão de processos de negócio e BPMN.

\subsection{Compreensão de Modelos}

A modelagem, no seu sentido mais amplo, é o uso econômico de algo (o modelo) no lugar de alguma coisa real, tendo em vista algum objetivo cognitivo [13]. Essa prática permite o uso de algo mais simples, seguro e com um menor custo do que o sistema real, para o estudo do objetivo desejado. Um modelo é, portanto, uma representação simplificada de algum conceito ou situação, com os objetivos de sua observação, manipulação e entendimento [14].

$\mathrm{Na}$ modelagem de processos, tal como em outras aplicações, os modelos são criados com o objetivo de diminuir a complexidade inerente aos temas de suas aplicações [13]. A utilização de modelos é uma forma de resolver a limitada capacidade cognitiva dos seres humanos visto que, em geral, há uma certa dificuldade em se compreender um ambiente complexo em sua totalidade [15]. Assim, a única maneira de compreendê-lo é através de um modelo simplificado, que ressalta alguns aspectos do ambiente em detrimento de outros.

Segundo Reijers e Mendling [3], o termo compreensão se refere à capacidade de entender as informações contidas em um modelo. Todavia, essa definição implica que a compreensão pode ser investigada a partir de dois 
ângulos principais: fatores pessoais, relacionados ao leitor do modelo e os fatores que se relacionam com o modelo propriamente dito. Corroborando com essa definição, Mendling, Strembeck e Recke [1] destacam que a compreensão de um modelo de processo é uma função relacionada com as características do modelo e com os usuários que vão interpretar o modelo. Para estes autores a compreensão dos modelos pelos stakeholders é um pré-requisito para várias tarefas relacionadas ao modelo, tais como: comunicação, desenho, reengenharia organizacional, gestão de projetos, consultas dos usuários finais etc. De acordo com Figl e Laue [16], uma pessoa compreende um modelo de processo quando ela é capaz de explicar o modelo. Em outras palavras, há compreensão quando o usuário consegue explicar a estrutura, o comportamento e os efeitos do modelo sobre o seu contexto.

\subsection{Gestão de Processos de Negócio}

A Gestão de Processos de Negócio (do inglês, Business Process Management ou BPM) é um conceito que une gestão de negócios e tecnologia da informação para garantir resultados consistentes e aproveitar as oportunidades de melhoria para uma organização. Ou seja, descreve a gestão de cadeias inteiras de eventos, atividades e decisões que agregam valor para a organização e seus clientes. Essas cadeias de eventos, atividades e decisões são chamadas processos [17]. Nesse contexto, um processo pode ser definido como um conjunto estruturado de atividades ordenadas no tempo e no espaço, que produz um produto ou um serviço, com início e fim, e com entradas e saídas bem definidas [18].

Segundo Dumas et al. [17], o BPM envolve um ciclo de vida com as seguintes fases: (i) identificação do processo - atividade em que os processos organizacionais são identificados, delimitados e criticados quanto há problemas, deficiências ou oportunidades de melhoria; (ii) descoberta dos processos - atividade em que os processos são modelados na forma como são executados na organização (AS-IS) e documentados; (iii) análise dos processos - atividades em que os processos são avaliados quantitativa ou qualitativamente em relação ao seu desempenho; (iv) redesenho de processos - atividade que ocorre as alterações necessárias que ajudariam a resolver os problemas identificados na fase anterior e permitir que a organização cumpra os seus objetivos de desempenho são projetadas em um novo modelo de processo (TO-BE); (v) implementação do processo atividade em que os processos redesenhados são implementados na organização, o que necessariamente envolve treinamento e, na maioria das vezes, automação; (vi) monitoramento e controle dos processos - atividade responsável em coletar os dados dos processos em execução para avaliar se o desempenho atende as necessidades previstas inicialmente.

Neste contexto, processos são tratados como uma coleção inter-relacionada de atividades de trabalho, iniciadas em resposta a um evento, alcançando um resultado específico para um cliente e outros patrocinadores do processo [19]. Dessa forma, busca-se a partir dos modelos de processo de negócio representar um conjunto de elementos de negócio e suas relações necessárias para atingir um objetivo organizacional.

Um modelo de processo de negócio é uma representação do conjunto de atividades de uma organização, com uma estrutura que descreve sua ordem lógica e dependência [13]. Dentre as diversas técnicas e abordagem utilizadas para representar modelos de processos, o BPMN é o de maior destaque [20]. A próxima seção apresenta um breve histórico da notação BPMN, destacando os seus principais artefatos, de acordo com [21].

\subsection{Business Process Model and Notation}

BPMN foi desenvolvido em 2004 como uma especificação aberta para a modelagem de processos de negócio, sendo incorporado, já em 2006, ao Grupo de Gestão de Objetos (do inglês: Object Management Group ou OMG). Seu objetivo principal é fornecer uma notação, conforme já mencionado, que pudesse ser facilmente utilizada e compreendida por todos os usuários da organização, desde os analistas de negócios, que esboçam os primeiros desenhos dos processos, passando pelos técnicos e desenvolvedores responsáveis da implementação, e finalmente os líderes que gerenciam e acompanham os processos [22].

\subsubsection{Artefatos mais utilizados}

O BPMN é uma notação robusta que apresenta uma ampla gama de elementos que podem ser agrupados em cinco categorias básicas descritas a seguir, de acordo com a OMG [5]: 
1. Objetos de fluxo: são os elementos mais básicos que definem o comportamento do processo de negócio. Estes elementos se dividem em três categorias: eventos, atividades e gateways;

2. Objetos de conexão: são os responsáveis por estabelecer a conexão entre os objetos de fluxo através de diferentes tipos de setas. Os objetos de conexão se dividem em quatro categorias: fluxo de sequência, fluxo de mensagens e associação;

3. Swimlanes: auxiliam a agrupar e a organizar os elementos em categorias separadas para diferentes responsabilidades funcionais. Podem ser catalogados em duas categorias: piscinas e raias;

4. Artefatos: são os elementos utilizados para fornecer informações adicionais aos processos (dados processados ou outros comentários). Esses elementos se dividem em duas categorias: grupo e anotação de texto;

5. Dados: são os elementos gráficos que representam tanto os itens físicos como as informações relativas à execução dos processos. Estes elementos se dividem em seis categorias: objeto de dados, armazenamento de dados, dados de entrada, dados de saída, conjunto de entrada e o conjunto de saída.

Tendo como objetivo prover recomendações específicas para o grupo de modeladores de processos de negócios, zur Mühlen e Recker [23] realizaram uma pesquisa para identificar o conjunto mais comumente usados de elementos BPMN e como estes devem ser usados. Em sua pesquisa, os referidos autores examinaram 120 modelos BPMN obtidos de três fontes diferentes (websites, projetos de consultoria e seminários de BPMN), para os quais foram contabilizados os artefatos mais usados nos modelos. Assim, nesta pesquisa foi identificado que apenas um pequeno subconjunto de elementos BPMN são realmente utilizados na prática.

Uma análise mais recente é apresentada por Kunze et al. [24]. Os autores pesquisaram o uso dos artefatos mais usados dentro de 1.210 modelos BPMN criados na academia, na sua maioria por estudantes como parte de trabalhos de uma disciplina. Os resultados apontam similitude com os que outrora foram obtidos por zur Mühlen e Recker [23]. A maioria dos modeladores recorrem a apenas um subconjunto bastante limitado de vocabulário, usando como base sequências simples de atividade. De acordo com os resultados apresentados pelos estudos mencionados, apenas 14 artefatos da notação foram selecionados. A Tabela 1 apresenta esses artefatos com uma breve descrição de acordo com a OMG [5].

Tabela 1: Elementos BPMN selecionados.

\section{Elemento}

Descrição

Artefato

Fluxo de Sequência Utilizado para mostrar a ordem de execução dos objetos

(Sequence Flow) de fluxo no processo.

\begin{tabular}{cl}
$\begin{array}{c}\text { Atividades } \\
(\text { Task })\end{array}$ & $\begin{array}{l}\text { Elemento genérico que representa as ações realizadas } \\
\text { durante um processo. }\end{array}$ \\
\hline $\begin{array}{c}\text { Evento Final } \\
\text { End Event })\end{array}$ & Indica o final do processo. \\
\hline $\begin{array}{c}\text { Evento Final com } \\
\text { Mensagem } \\
\text { Message End Event })\end{array}$ & $\begin{array}{l}\text { Indica que uma mensagem é enviada a um dos } \\
\text { participantes na conclusão do processo. }\end{array}$ \\
\hline $\begin{array}{c}\text { Evento de Início com } \\
\text { Mensagem }\end{array}$ & $\begin{array}{l}\text { Indica o início do processo por meio da chegada de uma } \\
\text { mensagem de um dos participantes do processo. }\end{array}$ \\
$\begin{array}{c}\text { Piscina } \\
\text { (Pool })\end{array}$ & $\begin{array}{l}\text { Podem representar processos ou participantes externos } \\
\text { ao processo. }\end{array}$
\end{tabular}




\begin{tabular}{|c|c|c|c|}
\hline \multirow{2}{*}{$\begin{array}{l}\text { Raias } \\
\text { (Lanes) }\end{array}$} & \multirow{2}{*}{$\begin{array}{l}\text { Uma piscina pode ser subdividida em raias, onde cada } \\
\text { raia representa um participante interno do processo. }\end{array}$} & $\overline{\mathrm{a}}$ & \\
\hline & & 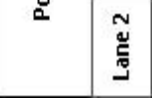 & \\
\hline $\begin{array}{l}\text { Gateway Exclusivo } \\
\text { (Exclusive Gateway) }\end{array}$ & $\begin{array}{l}\text { Denota situações em que apenas uma atividade pode ser } \\
\text { ativada. }\end{array}$ & & \\
\hline $\begin{array}{l}\text { Gateway Paralelo } \\
\text { (Parallel Gateway) }\end{array}$ & $\begin{array}{l}\text { Denota situações em que as atividades devem ser } \\
\text { realizadas em paralelo. }\end{array}$ & & \\
\hline $\begin{array}{l}\text { Gateway Inclusivo } \\
\text { (Inclusive Gateway) }\end{array}$ & $\begin{array}{l}\text { Denota situações em que as atividades podem ser ou não } \\
\text { realizadas em paralelo. }\end{array}$ & & \\
\hline $\begin{array}{l}\text { Evento Intermediário } \\
\text { com Mensagem } \\
\text { (Message Intermediate } \\
\text { Event) }\end{array}$ & $\begin{array}{l}\text { Contempla o envio de uma mensagem para um dos } \\
\text { participantes do processo. }\end{array}$ & & \\
\hline $\begin{array}{l}\text { Evento Intermediário } \\
\text { com Tempo } \\
\text { (Timer Intermediate } \\
\text { Event) }\end{array}$ & $\begin{array}{l}\text { Pode ser assinado um período de tempo para acionar um } \\
\text { evento. }\end{array}$ & & \\
\hline $\begin{array}{l}\text { Fluxo de Mensagens } \\
\quad \text { (Message Flow) }\end{array}$ & $\begin{array}{l}\text { Representa a comunicação entre dois participantes que } \\
\text { estão aptos para troca de mensagens. O fluxo de } \\
\text { mensagem é frequentemente utilizado para indicar a } \\
\text { comunicação entre processos. }\end{array}$ & o- -- & $----\rightarrow$ \\
\hline $\begin{array}{l}\text { Objetos de Dados } \\
\text { (Data Object) }\end{array}$ & $\begin{array}{l}\text { Fornecem informações adicionais sobre o que o processo } \\
\text { faz, não alterando se comportamento. }\end{array}$ & & $\Delta$ \\
\hline
\end{tabular}

Tendo como base essas duas pesquisas realizadas por zur Mühlen e Recker [23] e Kunze et al. [24], a presente pesquisa optou por verificar a compreensão desses artefatos, seguindo os procedimentos metodológicos que estão apresentados na próxima seção.

\section{Procedimentos Metodológicos}

Para garantir o nível de cientificidade do estudo é importante explicitar o procedimento metodológico que viabilizou a chegada até o conhecimento. Ou seja, exibir o conjunto de procedimentos e técnicas adotado para alcançar o entendimento esperado [25]. De início, há algumas menções a respeito do método de pesquisa, que é um procedimento delineador que apoia o modo indutivo de encarar a pesquisa, ressaltando a importância da interpretação da complexidade de uma situação [26] e provê diferentes dimensões para uma investigação com rigor científico [27].

Por opção, ao se centrar na objetividade, considerando que a realidade pode ser compreendida com base na análise de dados brutos, buscando indicadores e tendências factíveis de observação, soa como natural a escolha de um método quantitativo para esta pesquisa. Justifica-se a escolha de um método de pesquisa quantitativo, pois permitiu a análise e validação dos dados obtidos durante o processo do experimento, os quais se mostraram importantes para comprovar o nível de compreensão dos participantes com a notação BPMN.

Esta seção apresenta os procedimentos do experimento com as questões de pesquisa, os participantes do experimento, os instrumentos e os procedimentos utilizados para verificar a compreensão do processo de negócio entre uma representação textual e uma representação com um modelo BPMN. 


\subsection{Questões de Pesquisa}

O objetivo desta pesquisa foi verificar se um determinado cenário de negócio é mais compreensível para usuários da área de gestão se ele estiver representado em texto ou em um modelo BPMN. Dessa forma, foram definidas as seguintes questões de pesquisa:

- RQ1: BPMN é de fácil compreensão, se o conhecimento dos participantes na notação não for considerado?

- RQ2: BPMN é de fácil compreensão, se o conhecimento dos participantes na notação for considerado?

A ideia é verificar se há diferenças na compreensão de um processo que é retratado em uma linguagem familiar (texto) versus uma representação gráfica - BPMN - que alguns autores indicam ser de fácil compreensão. Assim, será possível verificar a veracidade de alguns autores (Tian et al. [6]; Martínez-Salvador et al. [7]; Istoan [8]; Geambasu [9]; Fernández et al. [10]; Schrepfer et al. [11]; Wahl e Sindre [12]), que ressaltam o fato de BPMN ser uma notação intuitiva e de fácil compreensão para os seus usuários.

\subsection{Participantes}

Ao avaliar como os leitores compreendem uma notação, é importante identificar a população de leitores que está sendo considerada [28]. Aqui foi considerado como leitores somente os gestores de negócio, uma vez que eles devem analisar os modelos de processos de negócios em uma organização. Segundo De Sordi [29], os gestores de negócio devem ter plena responsabilidade e autoridade sobre os seus respectivos processos de negócios, e isso engloba:

- Assegurar os recursos necessários para atender às demandas do processo de negócios conforme seu projeto em vigor;

- Realizar a medição contínua do desempenho de aspectos críticos do processo;

- Assegurar a capacitação dos profissionais que cooperam com o processo por meio de programas de treinamento;

- Definir e coordenar as alterações necessárias para a evolução contínua do processo.

Sendo os gestores de negócio também considerados os donos dos processos de negócios, eles devem ter o mínimo necessário de compreensão a respeito da notação utilizada para a modelagem dos processos. Assim, optou-se em realizar a pesquisa com participantes no início da carreira, com pouca ou nenhuma experiência na notação BPMN. O objetivo por essa escolha foi evitar qualquer tipo de víeis em relação aos participantes (treinamento especializado ou coisas do gênero), pois foi presumido que esse tipo de profissional, mesmo afirmando que conhecia a notação, não fosse de fato um especialista nela. Logo, com esse perfil, foram selecionados 94 participantes.

\subsection{Instrumentos e Procedimento}

Nesta investigação, optou-se por utilizar três instrumentos: uma descrição textual de um cenário, a representação desse mesmo cenário em BPMN e um questionário para avaliar a compreensão do contexto com algumas perguntas específicas em relação ao cenário apresentado. Logo, se optou em dividir o grupo de participantes em dois: um apenas para trabalhar com a descrição textual do negócio (47 participantes) e o outro com o modelo representado em BPMN (47 participantes). Os participantes com conhecimento de notação BPMN foram assinalados aleatoriamente dentro dos dois grupos.

O cenário utilizado nesta verificação foi um processo do mundo real de uma empresa que fornece avaliações de imóveis. O cenário descreve que o processo deveria iniciar com o envio de uma solicitação de avaliação do imóvel por parte do proprietário. Após o registro da solicitação, com o cadastramento do imóvel e do proprietário pelo setor de atendimento (que devem ser executados concomitantemente), a avaliação do imóvel é agendada e a documentação encaminhada ao setor jurídico para verificação. Caso a documentação esteja irregular, o processo é encerrado; caso contrário, a avaliação do imóvel é realizada pelo corretor conforme agendado. A Figura 1 apresenta a descrição do cenário representada na notação BPMN. 
Figura 1: Processo de Avaliação do Imóvel em BPMN.

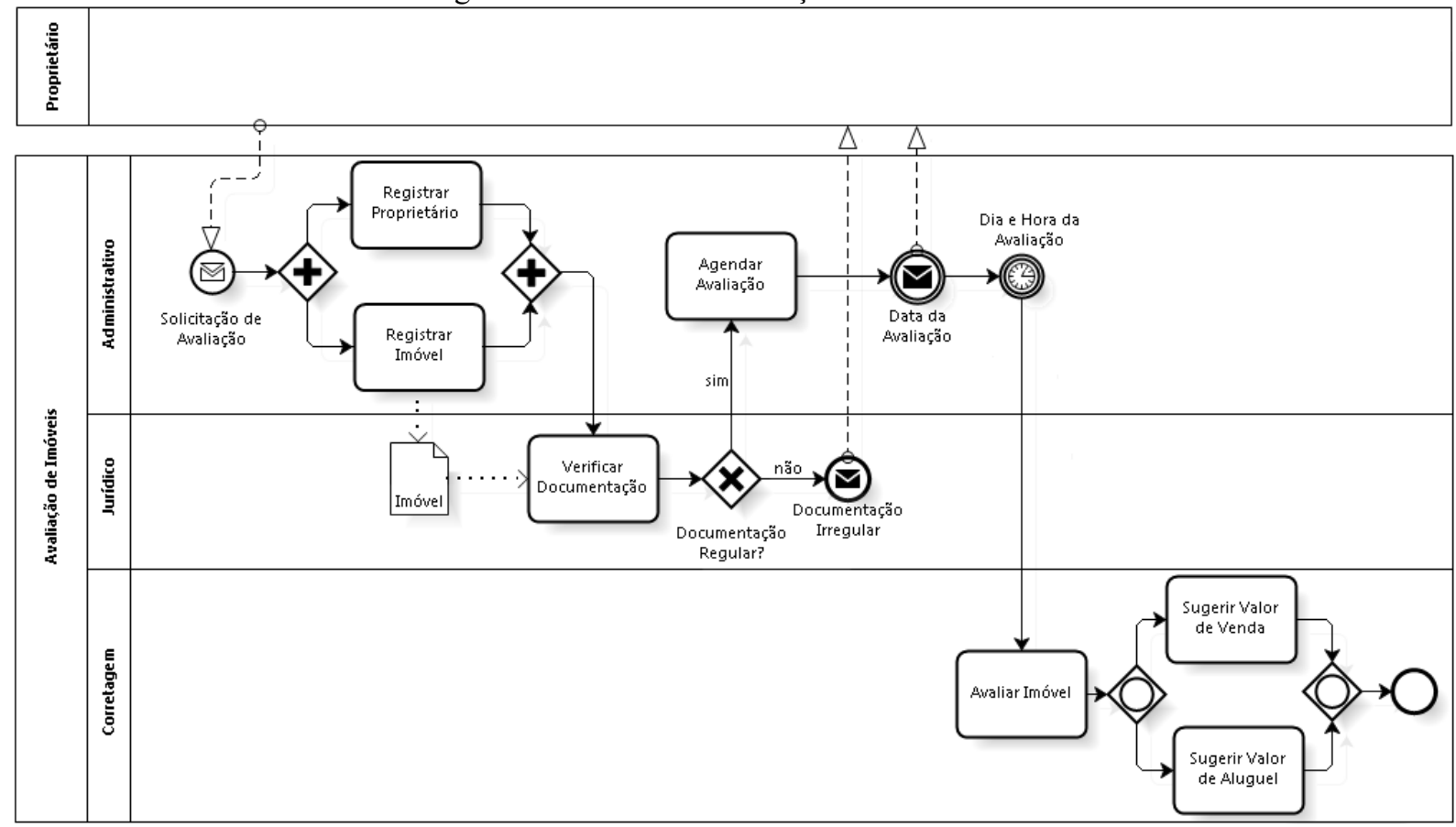

A escolha desse cenário se deu porque, além de representar um caso do mundo real, ele é relativamente pequeno, e, portanto, é compreensível em um curto espaço de tempo. Além disso, o cenário escolhido envolve um domínio que é compreensível para a maioria das pessoas que trabalham na área de gestão. Outro fator que é importante destacar é o fato de que a representação gráfica em BPMN (Figura 1) usou os artefatos mais utilizados na notação, conforme já mencionados. Cabe aqui ressaltar que para a aplicação dos instrumentos de pesquisa não foi colocado nenhuma restrição em relação ao tempo.

Um método comum para avaliar a compreensão dos modelos de processos de negócios é atribuir perguntas aos participantes sobre o conteúdo de um ou mais modelos e medir quão bem eles reconhecem um contexto [4]. O questionário utilizado, elaborado a partir da estrutura apresenta em [30], com os participantes foi composto por dez perguntas (Tabela 2) com declarações sobre o processo. No que se refere às possíveis respostas, foram apresentas três opções com apenas uma correta. As perguntas foram concebidas para avaliar a compreensão do cenário, independentemente do seu formato (textual ou BPMN).

Tabela 2: Perguntas sobre o processo.

\begin{tabular}{|c|c|c|}
\hline \# & Perguntas & Opções \\
\hline 1 & $\begin{array}{l}\text { O setor de Corretagem participa do } \\
\text { processo... }\end{array}$ & $\begin{array}{l}\text { a).... o o dia e hora da avaliação foi marcada. } \\
\text { b)...se a documentação do imóvel estiver irregular. } \\
\text { c)....se sugerir valor de venda. }\end{array}$ \\
\hline 2 & O setor de Jurídico é responsável por... & $\begin{array}{l}\text { a) ...agendar avaliação. } \\
\text { b) ...avaliar imóvel. } \\
\text { c) ...verificar documentação. }\end{array}$ \\
\hline 3 & $\begin{array}{l}\text { As atividades "Sugerir Valor de Venda" e " } \\
\text { Sugerir Valor de Aluguel", no setor } \\
\text { Corretagem... }\end{array}$ & $\begin{array}{l}\text { a) ...podem ambas serem executadas. } \\
\text { b) ....sempre somente uma será executada. } \\
\text { c) ....sempre as duas serão executadas. }\end{array}$ \\
\hline 4 & $\begin{array}{l}\text { Desde o início do processo, quantas } \\
\text { notificações um proprietário pode receber? }\end{array}$ & $\begin{array}{l}\text { a) Uma notificação. } \\
\text { b) Duas notificações. } \\
\text { c) Três notificações. }\end{array}$ \\
\hline 5 & Se a documentação não estiver regular... & $\begin{array}{l}\text { a) ...a avaliação é agendada. } \\
\text { b) ...é enviada uma notificação ao proprietário. } \\
\text { c) ...o processo é encerrado e é enviada uma } \\
\text { notificação ao proprietário. }\end{array}$ \\
\hline
\end{tabular}




\begin{tabular}{lll}
\hline 6 & As atividades "Registrar Proprietário" e & a) ...são sempre executadas. \\
& "Registrar Imóvel", no setor & b) ...apenas uma é executada. \\
& Administrativo... & c) ...pelo menos uma é executada. \\
\hline 7 & De quantas maneiras pode ser terminado o & a) Três maneiras. \\
& processo? & b) Uma maneira. \\
\hline 8 & O Proprietário participa do processo & c) Duas maneiras. \\
\hline & quando... & a) ... solicita uma avaliação. \\
& b) ...registra o imóvel. \\
& quantas atividades? & c) ...seleciona a data de avaliação. \\
\hline 10 & Que informações são necessárias para & a) Duas atividades. \\
& iniciar as atividades do setor jurídico? & b) Três atividades. \\
& c) Cinco atividades. \\
\hline
\end{tabular}

Todavia, a elaboração do questionário também buscou contemplar os artefatos mais utilizados, conforme apresentados por Kunze et al. [24] e zur Mühlen e Recker [23]. Assim, de forma mais individualizada, as questões da Tabela 2 têm uma correlação direta com respectivamente os seguintes artefatos BPMN: 1. Evento Intermediário; 2. Raias; 3. Gateway Inclusivo; 4. Fluxo de Mensagem; 5. Gateway Exclusivo; 6. Gateway Paralelo; 7. Evento Final; 8. Evento de Início e Piscinas; 9. Atividade e Fluxo de Sequência; e 10. Objetos de Dados.

\section{Análise e Discussão}

Esta seção apresenta os resultados a partir da análise dos dados coletados. Ela aborda a questão de pesquisa que foi definida, analisando também as partes do modelo BPMN que foram mais difíceis de entender. $\mathrm{Na}$ questão apresentada, o interesse era determinar se existe diferença na comparação das representações apresentadas entre: o número de respostas corretas dadas pelos participantes que receberam a descrição textual e o número de respostas corretas dadas por aqueles que receberam o modelo BPMN.

\subsection{Texto versus BPMN (sem considerar conhecimento em BPMN)}

Esta seção visa dar resposta à RQ1, analisando se existem diferenças estatísticas na comparação entre as respostas corretas dadas pelos participantes que receberam a descrição textual com o número de respostas corretas dadas por aqueles que receberam o modelo BPMN, independentemente do conhecimento prévio do BPMN.

Para esse propósito foi contabilizado para todos os participantes a quantidade de questões corretas, sendo assim possível realizar o teste de normalidade Shapiro-Wilk [31] para as duas amostras (representação textual e $\mathrm{BPMN}$ ) das respostas corretas. $\mathrm{O}$ resultado desse teste mostrou que nenhuma das duas amostras seguiram uma distribuição normal (o $p$-value foi menor que 5\%). Desta forma, foi aplicado o teste não-paramétrico MannWhitney [32]. O resultado do teste Mann-Whitney indicou uma diferença estatisticamente significativa entre as respostas, apresentando o p-value $=2.925 \mathrm{e}^{-06}$. O teste Vargha and Delaney [33] apresentou um tamanho de efeito $A_{12}=0.775$, sugerindo que aproximadamente $78 \%$ das vezes o entendimento do cenário com a representação textual foi melhor que o entendimento do modelo BPMN.

Assim, se não for considerado o conhecimento dos participantes em relação à modelagem de processos de negócios com BPMN, a representação textual vai proporcionar uma maior compreensão do processo de negócio em comparação a representação em BPMN. Isso induz a perceber que a descrição textual de um cenário, para esse grupo de participantes, é mais factível de compreensão que um modelo representado em BPMN, divergindo da literatura que, conforme já mencionado, ressalta a fácil compreensão da notação por ela ser intuitiva. As estatísticas descritivas desta seção estão resumidas na Figura 2, onde é possível observar graficamente as diferenças das respostas corretas obtidas pelos dois grupos (representação textual e BPMN). 
Figura 2. Número de respostas corretas por tipo de representação.

\begin{tabular}{|c|c|c|c|c|}
\hline \multicolumn{2}{|r|}{ Texto $\square$ BPMN } & \multicolumn{3}{|c|}{ Legenda } \\
\hline 10 & & & Texto $(n=47)$ & $\operatorname{BPMN}(n=47)$ \\
\hline \multirow{2}{*}{8} & & Valor minimo & 4.00 & 3.00 \\
\hline & & Prim eiro Quartil & 7.50 & 5.50 \\
\hline \multirow[t]{2}{*}{6} & & Mediana & 8.00 & 6.00 \\
\hline & & Média & 8.11 & 6.57 \\
\hline \multirow[t]{2}{*}{4} & & Terceiro Quartil & 9.00 & 8.00 \\
\hline & 1 & Valor máximo & 10.00 & 10.00 \\
\hline
\end{tabular}

\subsection{Texto versus BPMN (considerando conhecimento em BPMN)}

Esta seção tem como objetivo responder o RQ2, analisando se existem diferenças estatisticamente significativas entre as respostas corretas dadas pelos participantes nas diferentes representações (textual e BPMN), sendo eles do mesmo grupo. Assim, os dados, obtidos a partir do questionário (Tabela 2), foram também tabulados em uma tabela com quatro colunas. A primeira coluna identificando o participante, a segunda o tipo de representação recebida pelo participante (texto ou BPMN), a terceira se o participante conhece ou não a notação BPMN e, a última, o total de questões corretas. A Tabela 3 apresenta um fragmento dessa representação.

Tabela 3. Fragmento da tabela produzida a RQ2.

\begin{tabular}{cccc}
\hline $\begin{array}{c}\text { Número de } \\
\text { participante }\end{array}$ & $\begin{array}{c}\text { Tipo de } \\
\text { representação }\end{array}$ & Grupo & $\begin{array}{c}\text { Total respostas } \\
\text { corretas }\end{array}$ \\
\hline P1 & Texto & Conhece & 8 \\
\hline P2 & BPMN & Não conhece & 7 \\
\hline$[\ldots]$ & {$[\ldots]$} & {$[\ldots]$} & {$[\ldots]$} \\
\hline P93 & Texto & Conhece & 9 \\
\hline P94 & BPMN & Não conhece & 8 \\
\hline
\end{tabular}

Neste caso, a partir da tabela, ocorreram três tipos diferentes de análise. A primeira foi em relação aos participantes que conheciam BPMN, a segunda com os que não conheciam e a terceira uma comparação entre os dois grupos anteriores. As três seções subjacentes fazem uma análise sobre esses resultados.

\subsubsection{Grupo de participantes que conhecem BPMN}

A tabela 4 mostra um fragmento da tabela utilizada para realizar a análise entre os resultados apresentados pelo grupo de participantes que conhecem BPMN. O teste de normalidade Shapiro-Wilk [31] estabeleceu que os dois grupos (texto e modelo) seguiram uma distribuição normal ( $p$-value maior que 5\%). Logo, a homogeneidade de variância deve ser comprovada por meio do teste $F$ de Fisher [31]. O resultado do teste $F$ de Fisher aponta que existe igualdade nas variâncias dos grupos, valor de $p$-value $=0.3948$, maior que $5 \%$.

Dando continuidade, foi realizado o teste $t$ para amostras independentes [34], que foi usado para investigar se existem diferenças estatisticamente significativas entre os grupos. O $p$-value $=0.1138$, maior que $5 \%$, no teste $t$, indicou que não existe diferença estatisticamente significativa em relação às respostas corretas entre os grupos. Já o teste $g$ de Hedges [35] mostrou um tamanho de efeito de $g=0.58$.

Assim, é possível inferir que para os participantes que possuem algum conhecimento em modelagem BPMN, ambas as representações podem ser consideradas equivalentes. $\mathrm{O}$ resultado obtido pelo teste $t$, que não indicou diferença estaticamente significativa, sinaliza que há o mesmo nível de compreensão em relação ao entendimento do cenário descritos em texto ou em BPMN. 
Tabela 4. Fragmento da tabela para análise dos participantes que conhecem BPMN.

\begin{tabular}{ccccc}
\hline Grupo & $\begin{array}{c}\text { Número de } \\
\text { participante }\end{array}$ & $\begin{array}{c}\text { Total respostas } \\
\text { corretas BPMN }\end{array}$ & $\begin{array}{c}\text { Número de } \\
\text { participante }\end{array}$ & $\begin{array}{c}\text { Total respostas } \\
\text { corretas Texto }\end{array}$ \\
\hline Conhece & $\mathrm{P} 1$ & 9 & $\mathrm{P} 16$ & 8 \\
\hline Conhece & $\mathrm{P} 2$ & 5 & $\mathrm{P} 17$ & 7 \\
\hline Conhece & $\mathrm{P} 3$ & 10 & $\mathrm{P} 18$ & $6 \ldots]$ \\
\hline Conhece & {$[\ldots]$} & {$[\ldots]$} & {$[\ldots]$} & 10 \\
\hline Conhece & $\mathrm{P} 15$ & 9 & $\mathrm{P} 30$ & \\
\hline
\end{tabular}

\subsubsection{Grupo de participantes que não conhecem BPMN}

A tabela 5 mostra um fragmento da tabela utilizada para realizar a análise entre os resultados apresentados pelo grupo de participantes que não conhecem BPMN. O teste de normalidade Shapiro-Wilk [31] determinou que nenhum dos dois grupos (texto e modelo) seguiram uma distribuição normal ( $p$-value menor que 5\%). Depois, foi aplicado o teste não-paramétrico Mann-Whitney [32], com $p$-value $=4.374 \mathrm{e}^{-06}$, com resultado maior que $5 \%$, indicando que existe uma diferença estatisticamente significativa entre os dois grupos. Seguindo a análise, o teste Vargha and Delaney [33] mostrou um tamanho de efeito $A_{12}=0.828$, sugerindo que aproximadamente em $83 \%$ das vezes, o cenário descrito de forma textual dá um melhor resultado, se comparado ao cenário descrito em BPMN.

Assim, se os participantes não possuem conhecimento em BPMN, o cenário descrito em texto irá proporcionar uma maior compreensão do processo em comparação com ao modelo BPMN. Com isto, é possível inferir que para o uso de modelos em BPMN é necessário ter prévio conhecimento da sua semântica, mesmo com alguns autores (Tian et al. [6]; Martínez-Salvador et al. [7]; Istoan [8]; Geambasu [9]; Fernández et al. [10]; Schrepfer et al. [11]; Wahl e Sindre [12]) mencionando que o uso da notação é fácil.

Tabela 5. Fragmento da tabela para análise dos participantes que não conhecem BPMN.

\begin{tabular}{ccccc}
\hline Grupo & $\begin{array}{c}\text { Número de } \\
\text { participante }\end{array}$ & $\begin{array}{c}\text { Total respostas } \\
\text { corretas BPMN }\end{array}$ & $\begin{array}{c}\text { Número de } \\
\text { participante }\end{array}$ & $\begin{array}{c}\text { Total respostas } \\
\text { corretas Texto }\end{array}$ \\
\hline Não conhece & $\mathrm{P} 1$ & 10 & $\mathrm{P} 33$ & 8 \\
\hline Não conhece & $\mathrm{P} 2$ & 8 & $\mathrm{P} 34$ & 5 \\
\hline Não conhece & $\mathrm{P} 3$ & 9 & $\mathrm{P} 35$ & 6 \\
\hline Não conhece & {$[\ldots]$} & 7 & {$[\ldots]$} & {$[\ldots]$} \\
\hline Não conhece & $\mathrm{P} 32$ & {$[\ldots]$} & $\mathrm{P} 64$ & 7 \\
\hline
\end{tabular}

\subsubsection{Conhecem versus Não Conhecem BPMN}

Esta seção apresenta uma análise comparativa entre as respostas corretas e erradas, em um total de 940 respostas (94 participantes $\times 10$ questões), dos participantes que conhecem e não conhecem BPMN. A Figura 3 apresenta um gráfico que ilustra os acertos e erros dos grupos que conhecem e não conhecem BPMN, levando em consideração o tipo de representação (textual e BPMN). No gráfico é possível observar que a maior quantidade de respostas corretas foi obtida com a representação textual e, por outro lado, a maior quantidade respostas erradas foi dos participantes que estavam com a representação em BPMN. Logo, fica evidente que para os gestores a representação textual é mais conveniente, por maximizar a quantidade de acertos, quando os participantes foram arguidos em relação ao cenário aqui utilizado. 
Figura 3. Comparação das respostas de acordo com tipo de representação.

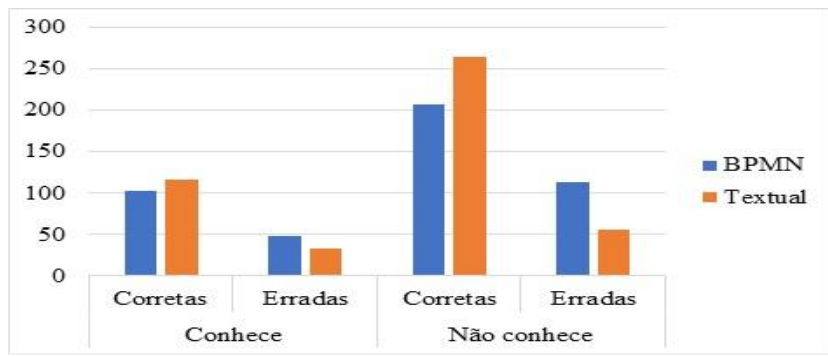

Na Figura 4 o gráfico foi dividido em quatro quadrantes (conhecem e acertaram, conhecem e erraram, não conhecem e acertaram e não conhecem e erraram), sendo possível visualizar que o melhor desempenho foi atribuído para os participantes com a representação textual. Uma outra questão que pode ser extraída do gráfico é o fato de os participantes que conhecem BPMN terem errado mais do que os participantes que também conhecem BPMN, mas responderam com o texto. Esse fato reforça o que já foi identificado; que o BPMN não é tão intuitivo e fácil de compreender como alguns autores já mencionados destacam.

Figura 4. Desempenho dos participantes de acordo com o conhecimento em BPMN.

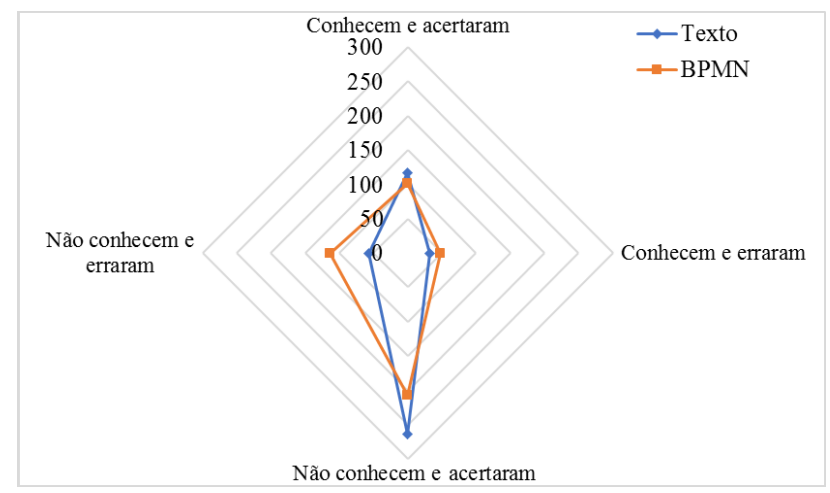

\subsubsection{Análise das questões sobre o processo}

Tendo como finalidade verificar de forma isolada todas as questões apresentadas no questionário (Tabela 2), foi calculado para todas as questões o percentual de acerto, apresentado na Figura 5. No gráfico da figura o eixo $y$ representa o percentual de acerto para cada questão e o eixo $x$ representa as questões. Deste modo, fica claro que a representação textual obteve uma compreensão melhor em oito questões. Todavia, da terceira questão até a oitava, o percentual de acerto foi superior a $15 \%$. O destaque para a representação em BPMN é apenas na questão 9 , onde ela obteve uma vantagem superior a $20 \%$.

Figura 5. Representação das respostas corretas para cada pergunta do processo.

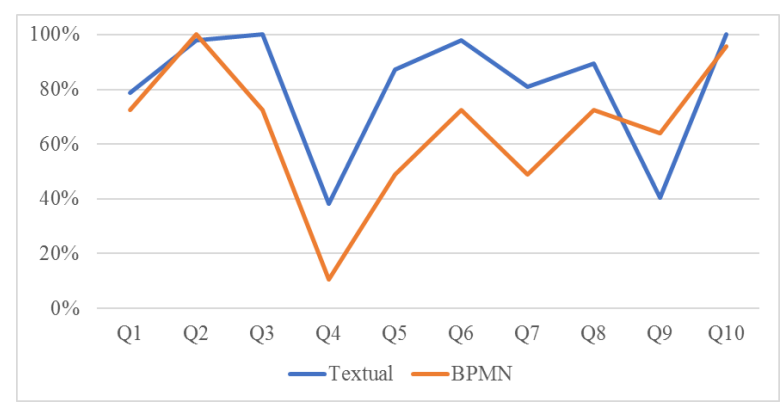




\subsubsection{Análise da compreensão dos elementos BPMN mais utilizados}

A Figura 6 apresenta o gráfico com os erros, em percentuais, para os artefatos já mencionados, para as respostas obtidas com a representação BPMN. No gráfico fica evidente que o artefato de fluxo de mensagens foi o que apresentou, com $89.36 \%$, um maior problema em relação à compreensão. Dando continuidade, os artefatos que seguem na lista com mais erros são: o gateway exclusivo e o evento final com o mesmo nível de erro $(51.06 \%)$, seguido por atividades e fluxo de controle (36.17\%).

Figura 6. Erros percentuais para as respostas com a representação BPMN.

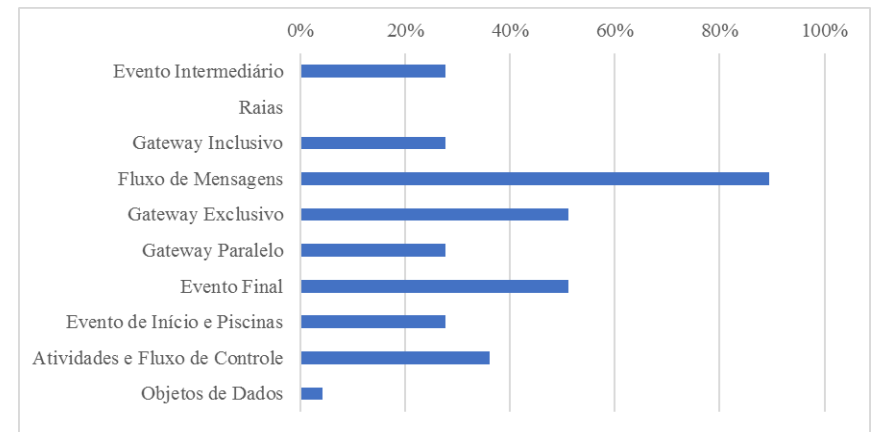

A Figura 7 faz o contraponto apresentando a quantidade de erros, também em percentual, para as respostas obtidas com a representação de texto. De forma equivalente à representação BPMN, o elemento com a percentagem com maior porcentagem de erros foi também o fluxo de mensagens $(61.70 \%)$. Os elementos atividades e fluxo de controle (59.57\%) e o evento intermediário (21.28\%) seguem essa lista em relação às respostas erradas.

Figura 7. Erros percentuais para as respostas com a representação textual.

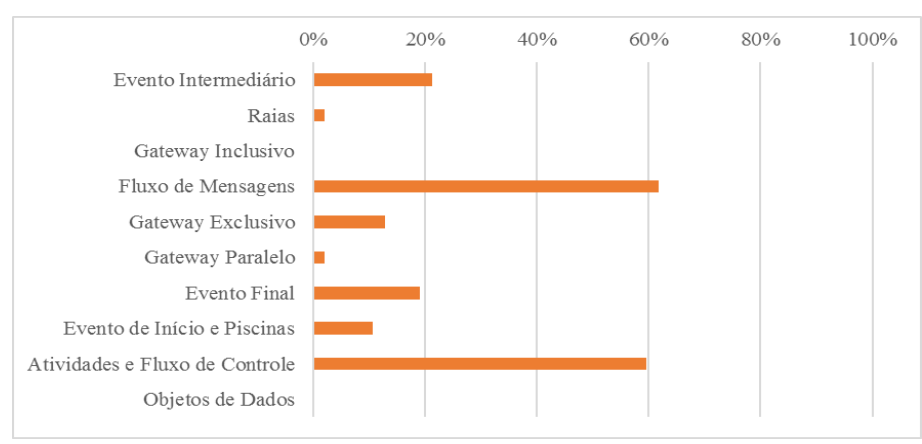

Assim, é possível concluir que a comunicação ou envio de notificações é um fator que gerou uma certa confusão nos participantes, acarretando em uma pouca compreensão do cenário apresentado. Essa evidência ficou mais notória com o elemento fluxo de mensagem na representação em BPMN. Porém, existem evidências que os gateways são conhecidos por afetarem a compreensão dos modelos em BPMN [30, 36], já que possuem muitas simbologias que impactam diretamente em sua compreensão. Por fim, cabe ressaltar que esta pesquisa foi realizada com pessoas da área de gestão, ou seja, são participantes que não são da área tecnologia. Doravante, essas pessoas vão, em algum momento, necessitar de algum conhecimento em relação aos modelos BPMN, que, conforme destaca Duarte [37], é a notação atualmente mais utilizada no mercado.

\section{Trabalhos Relacionados}

Existe uma extensa variedade de trabalhos que comparam diferentes notações de modelagem de processos de negócios com a representação BPMN com a finalidade de analisar a compreensão desses modelos, tais como:

- Rodrigues et al. [30] descrevem um experimento comparando modelos de processo textuais versus um modelo de processo de negócios em BPMN, tendo como resultado que o uso de representações textuais ou BPMN não influenciam a compreensão do processo para usuários não especializados, 
mas que influenciam aos usuários experientes. Porém, essa pesquisa não contemplou usuários da área de negócio;

- Ottensooser et al. [28] fizeram uma comparação entre a representação em BPMN com uma notação textual alternativa (baseado na descrição textual de casos de uso). Seus resultados mostraram aumentos significativos na compreensão do processo com o modelo textual. Todavia, isso não ocorreu para o modelo em BPMN, onde apenas os leitores treinados apresentaram aumentos significativos;

- Recker e Dreiling [38] apresentam um estudo experimental, comparando as representações em BPMN versus EPC (do inglês, Event-Drive Process Chain). Os autores sugerem que, caso o projetista conheça uma das duas linguagens apresentadas, eles provavelmente vão ter uma boa compreensão da outra linguagem;

- Figl [39] examinou como a escolha dos símbolos de uma notação de modelagem de processos de negócios pode afetar na compreensão de um contexto específico. $\mathrm{O}$ autor chegou à conclusão que não há apenas um melhor símbolo, mas que uma variedade de símbolos pode ser eficaz para promover a compreensão dos modelos para o usuário.

Cada um desses trabalhos realizou suas análises com pessoas que possuem algum grau de experiência com as notações. Assim, a presente pesquisa se difere das demais por usar participantes com pouca ou nenhuma experiência na representação BPMN; além dos participantes serem de um grupo específico de usuários: gestores de negócios.

\section{Considerações Finais}

Este artigo teve como objetivo verificar até que ponto jovens profissionais da área de gestão tem compreensão dos modelos de processos apresentados em BPMN quando comparados a representação textual. Avançando um pouco mais nessa discussão, Cross [40] sugere que o conhecimento de um projeto, aqui representado por um processo, reside especificamente no projetista, mas também pertence a todos que, de alguma forma, ajudam a projetar as soluções para o negócio.

Desta forma, foi realizada uma pesquisa quantitativa, com 94 participantes, para verificar o nível de compreensão dos jovens profissionais da área de gestão em relação à representação dos processos quando modelados em texto e em BPMN. Para tal, a pesquisa foi dividida em dois grupos de participantes: um para trabalhar com a descrição textual e o outro com o modelo representado em BPMN. Assim, foi utilizado como instrumento um questionário para avaliar a compreensão de um contexto a partir de algumas perguntas específicas em relação ao cenário apresentado. A partir das respostas obtidas, analisadas de forma estatística, foi possível constatar que os participantes desse grupo de pesquisa - os profissionais da área de gestão -, não possuem o conhecimento necessário para compreender modelos de processos representados em BPMN. Ou seja, os resultados evidenciam que para esse perfil de usuário a descrição de um cenário descrito em texto ainda proporciona uma maior compreensão quando comparado com representações em BPMN.

Tendo como premissa que é fundamental que todos os stakeholders, de gestão como de tecnologia, tenham a mesma compreensão dos modelos, a presente pesquisa ressalta como contribuição que isso ainda não é uma verdade. Logo, há indício que isso seja reflexo do pouco ou quase nenhum investimento em modelagem para o tipo de perfil de profissionais da área de gestão. Partindo do princípio que a modelagem de processos é amplamente usada no mercado, as instituições de ensino têm a responsabilidade de preparar seus estudantes, futuros profissionais, para essa competência.

Mas, por outro lado, ficou também evidente com os resultados que há uma necessidade de se equalizar o nível de compreensão dos artefatos de BPMN para que o uso dos mesmos possam realmente trazer benefícios às organizações. Ou seja, é interessante ressaltar para esse perfil de usuário a importância de se tornar um projetista a partir do que registrou Roger Martin, Reitor da Rotman School of Management [41]: "as pessoas de negócios não precisam só entender de melhor os designers; elas precisam se tornar designers". Por fim, como trabalho futuro, outra pesquisa experimental está sendo projetada para verificar se, para esse grupo de participantes, uma representação textual e uma representação BPMN (com um subconjunto de elementos da sua notação) podem de fato se complementar no contexto da modelagem de processos para facilitar realmente a compreensão.

Revista Brasileira de Computação Aplicada (ISSN 2176-6649), Passo Fundo, v. 9, n. 4, p. 60-75, dez. 201772 


\section{Agradecimentos}

Agradecemos o apoio financeiro da CAPES, através do Programa de Apoio à Pós-Graduação (PROAP), pelo qual este projeto está sendo financiado.

\section{Referências}

[1] MENDLING, J.; STREMBECK, M.; RECKER J. Factors of process model comprehension findings from a series of experiments. Decision Support Systems, Vol. 53, no. 1, 2012, pp. 195-206. Disponível em: $<$ http://www.sciencedirect.com/science/article/pii/S0167923612000085>. Acesso em: 30 nov. 2017.

[2] VANDERFEESTEN, I., et al. Quality metrics for business process models. BPM and Workflow handbook 144, 2007, pp. 179-190.

[3] REIJERS, H. A.; MENDLING, J.A. Study into the Factors That Influence the Understandability of Business Process Models. IEEE Transactions on Systems, Man, and Cybernetics-Part A: Systems and Humans, Vol. 41, no. 3, 2011, pp. 449-462.

[4] LAUE, R.; GADATSCH, A. Measuring the Understandability of Business Process Models-Are We Asking the Right Questions?. In: INTERNATIONAL CONFERENCE ON BUSINESS PROCESS MANAGEMENT, Springer Berlin Heidelberg, 2010, pp. 37-48.

[5] OMG. Object Management Group, Business process model and notation (BPMN) 2.0, 2011.

[6] TIAN, X. et. al. A Mapping Algorithm of Scientific Workflow from BPMN to BPEL. International Conference on Artificial Intelligence and Industrial Engineering- AIIE, 2015.

[7] MARTÍNEZ-SALVADOR, B.; MARCOS, M.; SÁNCHEZ, A. An Algorithm for Guideline Transformation: From BPMN to PROforma. Workshop on Knowledge Representation for Health-Care Data, Processes and Guidelines, 2014, pp. 121-132. Disponível em: <https://doi.org/10.1007/978-3-319-13281-5_9>. Acesso em: 30 nov. 2017.

[8] ISTOAN, P. Defining Composition Operators for BPMN. 11th International Conference Software Composition - SC, LNCS 7306, 2012, pp. 17-34. Disponível em: <https://doi.org/10.1007/978-3-64230564-1_2>. Acesso em: 30 nov. 2017.

[9] GEAMBASU, C.V. BPMN vs. UML Activity Diagram for business process modeling. Accounting and Management Information Systems, 11(4), 2012, p.637.

[10] FERNÁNDEZ, H.F. et. al. SBPMN - an easier business process modeling notation for business users. Computer Standards \& Interfaces, 32(1), 2010, pp.18-28. Disponível em: $<\mathrm{http}: / /$ www.sciencedirect.com/science/article/pii/S0920548909000300>. Acesso em: 30 nov. 2017.

[11] SCHREPFER, M. et. al. The impact of secondary notation on process model understanding. In: IFIP WORKING CONFERENCE ON THE PRACTICE OF ENTERPRISE MODELING, Springer Berlin Heidelberg, 2009, pp. 161-175.

[12] WAHL, T.; SINDRE, G. An analytical evaluation of BPMN using a semiotic quality framework. Advanced topics in database research 5, 2006, pp. 94-105.

[13] SILVEIRA, D. ANIMARE: Um Método de Validação dos Processos de Negócio Através da Animação. Tese de Doutorado, COPPE/UFRJ, Programa de Engenharia de Produção, Rio de Janeiro, 2009.

[14] MELLOR, J. S. et. al. MDA Distilled: Principles of Model-Driven Architecture, Addison-Wesley, ISBN: 0201-78891-8, 2004.

[15] PRESSMAN, R. S. Software Engineering: A Practitioner's Approach, 6 Edition, McGraw-Hill, ISBN: $0071238409,2004$.

[16] FIGL, K.; LAUE, R. Cognitive complexity in business process modeling. In: ADVANCED INFORMATION SYSTEMS ENGINEERING, 2011, pp. 452-466. 
[17] DUMAS, M. et. al. Introduction to Business Process Management, In: FUNDAMENTALS OF BUSINESS PROCESS MANAGEMENT, Springer, Berlin Heidelberg, 2013, pp. 1-31.

[18] LU, R,; SADIQ, S. A Survey on Comparative Modelling Approaches. 10th International Conference on Business Information Systems (BIS2007), Poznan, Poland, 2007. Disponível em: $<$ http://www.sciencedirect.com/science/article/pii/S1574119209000510>. Acesso em: 30 nov. 2017.

[19] SHARP, A.; MCDERMOTT, P. Workflow modeling: Tools for process improvement and application development, Norwood: Artech House, 2009.

[20] KOSSAK, F., et. al. A Rigorous Semantics for BPMN 2.0 Process Diagrams. Springer International Publishing, 2014, pp. 29-152.

[21] OMG. Business Process Model and Notation (BPMN), FTF Beta 1 for Version 2.0., 2009. Disponível em: $<\mathrm{http}$ ://www.omg.org $>$. Acesso em: 30 nov. 2017.

[22] WHITE, S. A. BPMN 1.0 business process modeling notation, OMG final adopted specification, on BPMN website, 2011.

[23] ZUR MUEHLEN, M.; RECKER, J. How much language is enough? Theoretical and practical use of the business process modeling notation. International Conference on Advanced Information Systems Engineering. Springer Berlin Heidelberg, 2008.

[24] KUNZE, M. et. al. Towards understanding process modeling-the case of the BPM academic initiative. In: INTERNATIONAL WORKSHOP ON BUSINESS PROCESS MODELING NOTATION, Springer Berlin Heidelberg, 2011, pp. 44-58.

[25] GIL, A.C. Métodos e Técnicas de Pesquisa Social. São Paulo: Atlas, 1989.

[26] CRESWELL, J.W. Projeto de Pesquisa Métodos Qualitativos, Quantitativo e Misto. Porto Alegre: Artmed, 2010.

[27] CHEN, W.; HIRSCHHEIM, R. A paradigmatic and methodological examination of information systems research from 1991 to 2001. Information Systems Journal, v. 14, n. 3, 2004, p. 197-235.

[28] OTTENSOOSER, A. et. al. Making sense of business process descriptions: An experimental comparison of graphical and textual notations. Journal of Systems and Software 85.3, 2012, pp. 596-606. Disponível em: $<$ http://www.sciencedirect.com/science/article/pii/S0164121211002408>. Acesso em: 30 nov. 2017.

[29] DE SORDI, J.O. Gestão por processos: uma abordagem da moderna administração. Saraiva, 2005.

[30] RODRIGUES, R.D.A. et. al. An experiment on process model understandability using textual work instructions and BPMN models. In: SOFTWARE ENGINEERING (SBES), 2015 29TH BRAZILIAN SYMPOSIUM IEEE, 2015, pp. 41-50.

[31] SPRENT, P.; SMEETON, N.C. Applied nonparametric statistical methods. CRC Press, 2016.

[32] ARCURI, A.; BRIAND, L. A practical guide for using statistical tests to assess randomized algorithms in software engineering. In: SOFTWARE ENGINEERING (ICSE), 2011 33RD INTERNATIONAL CONFERENCE, IEEE, 2011, pp. 1-10.

[33] VARGHA, A.; DELANEY, H.D. A critique and improvement of the CL common language effect size statistics of McGraw and Wong. JOURNAL OF EDUCATIONAL AND BEHAVIORAL STATISTICS, 25(2), 2000, pp.101-132.

[34] PITUCH, K.A.; WHITTAKER, T.A; STEVENS, J.P., Intermediate statistics: A modern approach. Routledge, 2015.

[35] HEDGES, L.V. Estimation of effect size from a series of independent experiments. Psychological bulletin, 92(2), 1982, p.490. Disponível em: <http://dx.doi.org/10.1037/0033-2909.92.2.490>. Acesso em: 30 nov. 2017.

[36] ROLÓN, E. et. al. Analysis and Validation of Control-Flow Complexity Measures with BPMN Process Models. Enterprise, Business-Process and Information Systems Modeling. Springer Berlin Heidelberg, 2009, pp. 58-70. 
[37] DUARTE R.B. et. al. Towards a Non-conformity Detection Method between Conceptual and Business Process Models. In: INTERNATIONAL CONFERENCE ON RESEARCH CHALLENGES IN INFORMATION SCIENCE, França, 2016.

[38] RECKER, J.C.; Dreiling, A. Does it matter which process modelling language we teach or use? An experimental study on understanding process modelling languages without formal education., 2007, pp. 356-366. Disponível em: <https://eprints.qut.edu.au/12270/1/68.pdf>. Acesso em: 30 nov. 2017.

[39] FIGL, KATHRIN. Symbol choice and memory of visual models. In: VISUAL LANGUAGES AND HUMAN-CENTRIC COMPUTING (VL/HCC), 2012 IEEE Symposium, IEEE, 2012, pp. 97-100.

[40] CROSS, N. Designerly Ways of Knowing. London: Springer Science \& Business Media, 2006.

[41] MARTIN, R. The design of business. Rotman Management 5(1), Winter, 2004, pp. 6-10. 